$1 \mathrm{P} 278$

\section{確率的シグナル伝達経路における外因性ノイズを含む入力信 号に対する応答性}

Responses of a stochastic signaling cascade to input signals with extrinsic noise

Akio Chiba ${ }^{1,2}$, Akihiro Fukagawa $^{1}$, Kumiko Sakata-Sogawa ${ }^{1,2}$, Makio Tokunaga ${ }^{1,2}\left({ }^{1}\right.$ Grad. Sch. Biosci. Biotech., Tokyo Inst. Tech., ${ }^{2} I M S-R C A I$, RIKEN)

Intracellular signaling cascades such as the MAPK pathway play an important role in amplification of input signals, notably faint signals. However, statistical fluctuations or noise in input signals are inevitable in vital signals. The signaling cascades amply not only signals but also noise, which often deteriorates the quality of the received signal. The properties of the deterioration in signaling cascades remain unclear. In this study, we performed the numerical simulations with a stochastic mathematical model of signaling cascade. We analyzed the effects of the extrinsic noise on the signal deterioration as a function of the parameters of input signals and cascades. We will discuss how living cells treat the extrinsic noise optimizing signaling cascades.
なぜ細胞は様々なステップ数を持つシグナルカスケードを使 いわけるのか?

Why do cells use signaling cascades with a variety of the number of steps?

Akihiro Fukagawa ${ }^{1}$, Masashi Kajita ${ }^{1}$, Kumiko Sakata-Sogawa ${ }^{1,2}$, Makio Tokunaga ${ }^{1,2}\left({ }^{1} \mathrm{Grad}\right.$. Sch. Biosci. Biotech., Tokyo Inst. Tech., ${ }^{2}$ IMS-RCAI, RIKEN)

Cellular signal transduction involves multiple steps, which are thought to be essential for signal intensity amplification. However, each reaction step adds an undesirable intrinsic noise to output signal. Why do living cells use such multistep processes? To answer this, we performed the numerical simulations based on a stochastic cascade model. We found signaling cascades maximize the signal-to-noise ratio at an optimal number of steps The number is logarithmically dependent on the input intensity, and is decreased with increasing the number of molecules at steps. This result indicates that cells select a signaling cascade system with an optimal number of steps and molecules according to the input signal intensity.

\section{Diffusion-controlled reaction rate-laws in intracellular} environment with molecular crowding: A single-particle-level simulation study

\section{Kazunari Kaizu, Koichi Takahashi (RIKEN)}

Although intracellular environment is significantly different from the ideal conditions expected in conventional biochemical simulations, the effect is poorly understood. In particular, there is no general quantitative theory for the reaction kinetics with molecular crowding. To derive the practical theory, we quantified the effect of molecular crowding on a rate-law in various conditions by using a single-particle-level simulation. In contrast to the common theory for diffusion-controlled reactions like CollinsKimball's, the recombination probability was evaluated as a kinetic parameter. The rate-law revised on the basis of these calculations allowed simulations of intracellular signaling pathways and revealed the impact of crowding on cellular responses.
$1 \mathrm{P} 282$

Power-law distribution derived from misunderstanding of search patterns

\section{Hisashi Murakami, Yukio Gunji (Kobe University)}

In the study of random search, though the Levy strategy is consistent with the searching behavior of real animals, some researchers claim that the Levy-like distributions exhibited by animals are not necessarily produced by a Levy process. Here, we propose an intermittent two-phase search model that does not include a Levy process, in which agent is basically a correlated random walker, but it memorizes its trajectory and counts the number of crossovers in a trajectory. If the number exceeds a threshold, the agent makes ballistic movement in the direction uncorrelated to the past. We show this model can optimize the trade-off. Finally, we demonstrate another model that misunderstand the rule to switch the two phases can show a Levy-like distribution of time intervals.
$1 \mathrm{P} 280$

\section{一分子シミュレーションによる上皮成長因子シグナル伝達経 路の応答不均一性の解明}

Understanding heterogeneity in EGF pathway using simulation at the molecular resolution

Kazunari Iwamoto, Yuki Shindo, Atsushi Miyauchi, Kazunari Kaizu, Koichi Takahashi (Laboratory for biochemical simulation, QBiC, RIKEN)

Cellular growth, proliferation and differentiation are regulated by epidermal growth factor (EGF) signaling pathway, and mutation of several proteins in EGF signaling pathway caused malignant transformation. In this pathway, ERK protein was finally activated and translocated into nucleus, which showed different dynamics between individual cells (called heterogeneity). Since heterogeneity affected the malignant transformation and drug resistance of cells, it is essential to elucidate the mechanism of heterogeneity. In this study, we simulate EGF signaling pathway at the molecular resolution and analyze the simulation results to understand mechanism of heterogeneity. Our simulation results showed one of mechanisms that can generate heterogeneity in EGF pathway.

\section{P283}

\section{From cell-autonomous circadian clocks to tissue-leve timekeeping}

Craig Jolley, Maki Ukai-Tadenuma, Dimitri Perrin, Hiroki Ueda (RIKEN Center for Developmental Biology)

In mammals, the suprachiasmatic nucleus $(\mathrm{SCN})$ is the central pacemaker of the brain. Each cell has an autonomous rhythm of gene expression, and $\sim 20,000$ neurons are coupled to produce a stable rhythmic output. We have developed a single-cell model of clock gene expression and parameterized it to experimental mRNA/protein expression data. We can predict the response of individual cells to perturbations, and we hope that these predictions will aid us in understanding intercellular coupling in the SCN. Currently, we are interested in how spatiotemporal "waves" of circadian gene expression arise at the tissue level. More coarse-grained models of the SCN will incorporate experimental data on intercellular coupling and help us understand organism-level behavioral outputs. 